\title{
COVID-19 and Obesity: Overlapping of Two Pandemics
}

\author{
Maria Alessandra Gammone Nicolantonio D'Orazio \\ Human and Clinical Nutrition Unit, Department of Medical, Oral and Biotechnological Sciences, University \\ G. D'Annunzio, Chieti, Italy
}

\section{Keywords}

Obesity · COVID-19 · Body fat · Pandemic · Inflammation .

Thrombosis

\section{Abstract}

Background: The coronavirus disease 2019 (COVID-19) pandemic has recently led to worldwide research efforts to identify subjects at greater risk of developing more severe illness: overall obesity displayed a strong correlation with critical illness and major severity of COVID-19 manifestations. Summary: Obesity and metabolic disorders are closely linked to chronic systemic inflammation. The adipose tissue constitutes a source of cytokines, which configure a low-grade inflammation and a hypercoagulation status; in addition, diagnosis and care of obese patients are often complicated by excess weight and ventilation difficulties. Key Messages: This review aims to examine the intersection between obesity and adverse outcomes of COVID-19, in order to investigate its preventive and/or therapeutic potential in the management of obesity-related COVID-19 complications.

(c) 2021 The Author(s).

Published by S. Karger AG, Basel

karger@karger.com www.karger.com/ofa

Karger $\stackrel{\text { ' }}{5}$

GOPEN ACCESS
C) 2021 The Author(s).

Published by S. Karger AG, Basel

This is an Open Access article licensed under the Creative Common Attribution-NonCommercial-4.0 International License (CC BY-NC) (http://www.karger.com/Services/OpenAccessLicense), applicable to the online version of the article only. Usage and distribution for commercial purposes requires written permission.

\section{Introduction}

Obesity is a fast-growing chronic disease of pandemic proportions with a current global prevalence of 39\%, whose complex pathophysiology is characterized by excessive hypertrophy and hyperplasia of adipose tissue due a chronic imbalanced energy state. Severe acute respiratory syndrome coronavirus 2 (SARS-CoV-2), responsible for COVID-19, has so far infected over 85 million and killed over 1.8 million people worldwide. The increased hospitalization and mortality linked to COVID-19 are associated with older age and a number of underlying conditions such as obesity and related comorbidities [1]. Obesity seems to increase the risk of both COVID-19 complications and mortality [2]. The increased risk of obese people to develop severe COVID-19 manifestations can be attributed to multiple potential mechanisms (Fig. 1), such as the chronic systemic phlogistic state, the dysfunctional and delayed immune response, and even the adipose tissue itself, which represents a reservoir for the virus.

It has been suggested that alveolar macrophages expressing angiotensin-converting enzyme 2 (ACE2) are the primary target cells for SARS-CoV-2 infection, and

Correspondence to:

Maria Alessandra Gammone, m.alessandra.gammone@gmail.com 


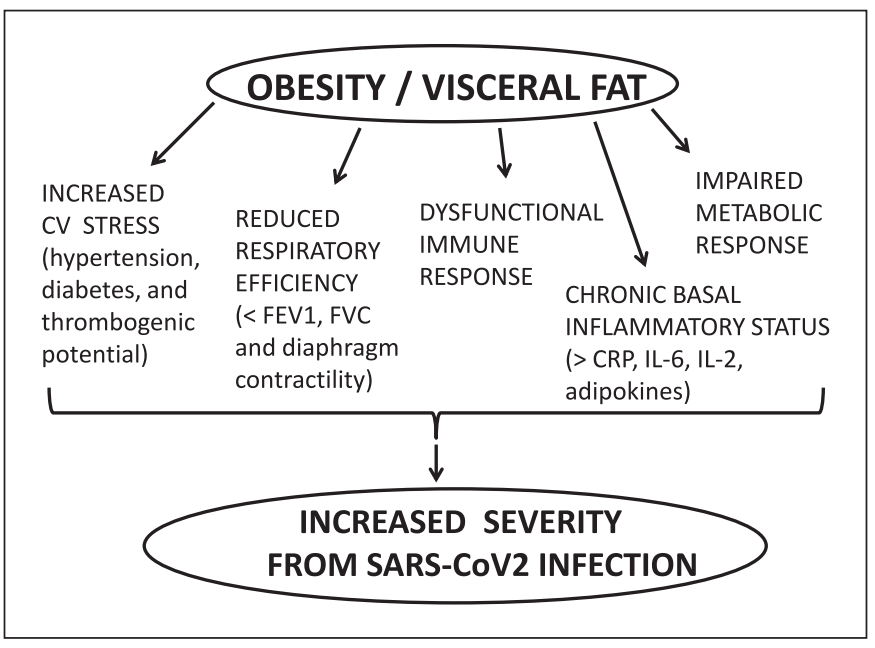

Fig. 1. Multiple pathways potentially linking obesity and visceral fat to increased severity from SARS-CoV-2 infection and COVID-19 disease. CV, cardiovascular; FEV1, forced expiratory volume; FVC, forced vital capacity; CRP, C-reactive protein; IL-6, interleukin 6 .

these activated macrophages may play a major role in the cytokine storm during COVID-19. SARS-CoV-2 gains entry into cells (with its transmembrane spike glycoprotein) through the ACE2-related carboxypeptidase receptor. The infection by SARS-CoV-2 likely eliminates the ACE2/angiotensin (Ang) regulatory axis leading to exaggeration of the ACE/Ang II/angiotensin type 1 receptor activity that directly facilitates ARDS and that is further enhanced by MHC-dependent antigen presentation and an aggressive inflammatory infiltrate [1]. The level of ACE2 expression in adipose tissue is higher than that in lung tissue; individuals with obesity have more adipose tissue, which ultimately translates into an increased number of ACE2-expressing cells and, consequently, a larger amount of ACE2, so that adipose tissue constitutes a reservoir for SARS-CoV-2 increasing the integral viral load [3]. Obesity is characterized by increased activation of the systemic and local adipose tissue renin-angiotensin system [4]: since the expression of several renin-angiotensin system components is increased in adipose tissue of people with obesity and Ang II has deleterious effects in multiple organs, it can be postulated that ACE2 in adipose tissue could provide a critical link between obesity and the susceptibility to COVID-19, resulting in more detrimental outcomes for obese patients.

Adipose tissue is also a source of many pro-inflammatory mediators and hormones. High baseline serum Creactive protein (CRP) and interleukin 6 (IL-6) levels, as well as hypoadiponectinemia and hyperleptinemia/leptin resistance (typical of obesity), elucidate the pre-existing inflammatory microenvironment in obese patients, making them more susceptible to worse outcomes and even fatality. Both excess adipose accumulation and lean mass loss (or their synergistic coexistence in case of sarcopenic obesity) can affect whole body functioning. These alterations may not only interfere with immunological responses to the virus but also exacerbate inflammatory reactions, worsening respiratory and metabolic distress; additionally, in the long term, they could influence time required for recovery, risk of long-term disabilities, and intensive care unit-acquired weakness, globally increasing the risk of critical illness and death.

\section{Mechanisms of Fat Inflammation in Obesity: Adipokine Dysregulation and Cellular Cross Talk}

Recent scientific evidence displayed a complex pathological framework around the deregulation of adipocytes that is not a simple diet-induced condition [5]. Adipose tissue represents an independent endocrine organ, able to store the energy provided by food. It releases a great amount of bioactive peptides, collectively named "adipokines," which play a central role in both immunity and vascular homeostasis.

Currently, it is well known that adipose tissue produces a huge variety of adipokines involved in the cardiovascular homeostasis, regulation of appetite, and glucose and lipid metabolism, among other biological functions. Adipokines have also numerous and complex involvements in the management of energy storage and employment and multiple cell metabolisms [6]. These molecules reach different organs and modulate a wide range of functions, from appetite control to inflammatory response [7] and can exert pro-inflammatory actions (such as leptin) or anti-inflammatory actions, such as adiponectin [8].

Leptin is the leading adipokine, whose anorexigen activity modulates satiety and food intake; its blood levels are proportionate to the amount of white adipose tissue and BMI. Leptin is an essential pulsatile hormone, responsible for energy homeostasis and numerous neuroendocrine functions [9]. It stimulates the migration of resident macrophages in the WAT and promotes their shift toward a pro-inflammatory profile and unbalances lymphocyte Th profiles, by reducing regulatory $\mathrm{T}$ cells and inducing Th17 polarization [10]. The typical modern diet-induced obesity is characterized by hyperleptinemia (elevated levels of leptin) and resistance to the body 
weight-reducing effects of leptin [11]. Leptin resistance upsets the endothelial leptin signaling, predisposing to atherogenesis in obese subjects, and it is responsible for the pro-inflammatory microenvironment, often leading to cardiovascular complications [12].

Adiponectin is another adipokine, whose blood level correlates negatively with the amount of adipose tissue [13]: it increases in proportion to subcutaneous fat but decreases with visceral fat accumulation. Leptin promotes insulin sensitivity and fatty acid oxidation and reduces hepatic neoglucogenesis [14]: low adiponectin levels are associated with numerous obesity-related metabolic diseases [15]. It is an anti-inflammatory hormone, inversely linked to CRP and IL-6 levels in obese individuals: low adiponectin levels are associated with elevated inflammatory mediators. Adiponectin promotes lymphocyte Th1 polarization and antiviral inflammation. Other adipokines, such as lipocalin-2, downregulate inflammatory $\mathrm{Th}$ polarization and regulatory lymphocytes. Clinical studies displayed a significant reversal of adiponectin, CRP, and IL-6 levels after bodyweight reduction in obese individuals [16]. This reciprocal interrelation of pro-inflammatory and anti-inflammatory adipokines and their imbalance contribute to the development of obesity-related metabolic and cardiovascular disorders [17-19]. Adipokines, involved in the pathogenesis of inflammation and insulin resistance, are overproduced with increasing adiposity and dysregulated in obesity (anti-inflammatory adipokines, such as adiponectin, decrease and pro-inflammatory ones, such as TNF- $\alpha$, and IL-6, increase), thus promoting metabolic complication. Two signaling pathways are activated by the pro-inflammatory cytokines in obesity: the nuclear factor-kB (NF-kB) and the c-Jun NH2-terminal kinase (JNK) pathway, which downregulate the antiinflammatory transcription factors thus propagating inflammatory processes [20]. Adipocyte hypertrophy is linked to altered intracellular signaling: enlarged omental adipocytes resulted to be hyperresponsive to TNF- $\alpha$ with an increased constitutive NF-kB activity, thus leading to adipokine overproduction [21]. Another crucial step in increased obesity-related inflammation is the massive infiltration of adipose tissue by phogosis mediators: monocyte chemoattractant protein-1 (MCP-1), whose circulating levels are elevated in obese, recruits monocytes and macrophages into the adipose tissue as well as into the arterial vessel wall. This process can contribute to cardiovascular events, which represent a frequent complication in COVID-19 patients. The cross talk between different cells and the molecular mediators of adipokine overproduction act synergistically. Additional mechanisms, such

COVID-19 and Obesity: A Fatal Overlapping as oxidative stress [22] and hypoxia, seem to have a role in the obese pro-inflammatory microenvironment. Adipose tissue hypoxia seems to be due to hypoperfusion of the rapidly expanding fat mass, where oxygen partial pressure and blood flow decrease [23]. This local hypoxia decreases the mRNA levels of adiponectin, while increasing those of pro-inflammatory genes (PAI-1, TGF, TNF- $\alpha$, IL- 1 , IL6 , and MCP-1) as well as those of hypoxia response genes (HIF-1, glucose transporter 1, and VEGF): these events exacerbate the inflammatory response in adipose tissue and contribute to obesity-related complications. Unlike subcutaneous fat, whose expansion is hyperplasic and not linked to low-grade inflammation [24], visceral fat accumulation (the so-called "abdominal obesity" or "central obesity") is usually marked by an impaired profile of adipokines, with increased pro-inflammatory signals. Most adipokines with pro-inflammatory properties (such as leptin, resistin, osteopontin, and chemerin) are overproduced with increasing adiposity, while others with antiinflammatory or insulin-sensitizing properties (such as adiponectin, ghrelin, and lipocalin-2) are decreased.

A further relevant group of adipokines is represented by acute phase reactants (CRP, serum amyloid A, plasminogen activator inhibitor-1, and haptoglobin) and damage-associated molecular pattern molecules (alarmin tenascin C, calprotectin, heat shock proteins, and HMGB1) that enhance the inflammatory status typical of obesity [25]. This dysregulation of adipokine secretion promotes inflammatory responses, metabolic dysfunction, and subsequent obesitylinked complications. Additionally, an impairment of the immune system may be present due to the dysregulation of the factors produced by adipose tissue. The trigger of this dysfunction could be the metabolic stress due to both nutrient excess and hypoxic stress determined by hypertrophic visceral adipocytes because of increased cell size and low neovascularization [26], via a mobilization of hypoxia-inducible factor 1 . The chronic low-grade inflammatory state in obesity has been confirmed by high baseline CRP in overweight individuals $[27,28]$. A Korean multicentric study evaluated the correlation between high-sensitivity CRP and sarcopenic obesity: CRP resulted to be significantly higher in obese patients in comparison with the normal weight control group [29].

\section{Endothelial Activation and Prothrombotic Substrate}

COVID-19 is associated not only with dysregulated inflammation but also with augmented coagulation and thrombotic accidents, which were extensively found in 
infected patients [30]. In numerous ARDS patients, venous thromboembolism and thrombocytopenia, renal failure, and disseminated intravascular coagulation have often been reported. In situ thrombi were found in pulmonary arteries and in other organs, including the liver and kidneys, in subjects who died of COVID-19 [31]. A systematic literature review [32] included 151 autopsies (age range was between 27 and 96 years), 91 of which presented microthrombi: $73 \%$ in the lung, $24 \%$ in the kidney, $16 \%$ in the liver, and $11 \%$ in the heart. The patients with microthrombi had more comorbidities such as arterial hypertension (62\%), obesity or overweight (64\%), cardiovascular disease (53\%), and diabetes mellitus type 2 (51\%). The most common histopathological modifications found in patients with lung microthrombosis were diffuse alveolar damage in exudative, pulmonary embolism, and lung infarct. These results show that microthrombi in COVID-19 autopsies can be found (especially in presence of comorbidities) in different organs. These autopsy findings propose the hypothesis that widespread endothelial hyperactivation in COVID-19 can trigger thrombotic events, with intra-alveolar deposits of fibrin and activated leukocytes contributing to respiratory failure [33]. The damage/hyperactivation of endothelial cells results in intracellular signaling pathways leading to the generation of cell adhesion molecules (circulating soluble intercellular/vascular adhesion molecules and E-selectin are increased in obese adults and decreased after weight loss), adipokines, and pro-inflammatory cytokines, which address inflammatory cells to the endothelium and underlying tissues [34]. The enhanced adhesion of monocytes to the endothelium and diapedesis of macrophages through the endothelial cell junctions into the adipose tissue [35] can be directly involved in thrombosis. Additionally, both endothelium and adipose tissue generate plasminogen activator inhibitor-1, whose increased levels (mostly induced by TNF- $\alpha$, thrombospondin-1, and oxidative stress) typical of obesity can determine hypofibrinolysis, thus configuring a prothrombotic state [36] and contributing to the poor prognosis of these patients $[37,38]$.

\section{Clinical Course and Outcome among Obese COVID-19 Patients}

In a study involving $>3,000$ COVID-19 patients, with 307 obese subjects younger than 60 years, individuals with a BMI between 30 and $34 \mathrm{~kg} / \mathrm{m}^{2}$ were 2 times more likely to be admitted to acute care and 1.8 to be admitted to critical care for ARDS compared with those with a BMI below $30 \mathrm{~kg} / \mathrm{m}^{2}$. Likewise, younger patients with a BMI of $35 \mathrm{~kg} / \mathrm{m}^{2}$ or higher were 2.2 times more likely to be admitted to acute and 3.6 to be admitted to critical care than patients in the same age category who had a BMI below $30 \mathrm{~kg} / \mathrm{m}^{2}$ [39]. Consistently, a study evaluating 781,000 young adults aged 18-34 years hospitalized with COVID-19 found a substantial rate of adverse outcomes: $21 \%$ required intensive care, $10 \%$ required mechanical ventilation, and $2.7 \%$ died. This in-hospital mortality rate is lower than that reported for older adults with COVID-19, but approximately double than that of young adults with acute myocardial infarction. Severe obesity, hypertension, and diabetes were common and associated with greater risks of adverse events. Young adults with $>1$ of these conditions faced risks comparable with those observed in middle-aged adults without them [40]. Similar results are available in an older population with a mean age of 60 years [41], were $47 \%$ of subjects admitted to the ICU had a BMI above $30 \mathrm{~kg} / \mathrm{m}^{2}$, whilst $28 \%$ of them were above $35 \mathrm{~kg} / \mathrm{m}^{2}$. The proportion of patients who required invasive mechanical ventilation increased with BMI categories, and it was the greatest in patients with a BMI above $35 \mathrm{~kg} / \mathrm{m}^{2}$ [41].

A recent retrospective cohort study [42] examined the correlation between different adiposity indexes and chest X-ray severity score in 215 COVID-19 hospitalized subjects and evidenced higher chest X-ray severity scores and higher rates of chest X-ray severity scores in individuals with abdominal obesity in comparison with patients without abdominal obesity. Waist circumference and waist-to-height ratio correlated more directly with chest $\mathrm{X}$-ray severity scores than BMI: a multivariate analysis indicated abdominal obesity, bronchial asthma, and oxygen saturation at admission as independent factors linked to high chest X-ray severity scores. Therefore, waist circumference should also be measured in SARS-CoV-2-infected subjects, and those with abdominal obesity should be closely monitored.

Another very recent international multicenter retrospective meta-analysis extracted data from health care records and regional databases of hospitalized adult patients with COVID-19 from 18 sites in 11 countries [43]. The odds of outcomes, such as supplemental oxygen/ noninvasive ventilatory support, invasive mechanical ventilatory support, and in-hospital mortality, were modeled by the BMI category, adjusting for age, sex, and prespecified comorbidities. Among over 7000 patients (65.6\% overweight/obese), those with overweight were more likely to require oxygen/noninvasive ventilatory support and invasive mechanical ventilatory support. 
There was no association between overweight and inhospital mortality. Similar effects were observed in patients with obesity or diabetes. According to these results, in adults hospitalized with COVID-19, overweight, obesity, and diabetes were associated with increased odds of requiring respiratory support; however, in patients with diabetes, the odds of severe COVID-19 were not increased above the BMI-associated risk.

More specifically, overweight women with polycystic ovary syndrome (PCOS) have recently been highlighted as an overlooked and potentially high-risk population for COVID-19 complications. A population-based study [44] identified 21,292 women with a coded diagnosis of PCOS and randomly selected 78,310 aged and general practice matched control women. Results revealed a 52\% increased risk of COVID-19 infection in women with PCOS, which remained increased at $28 \%$ above controls after adjustment for age, BMI, and impaired glucose regulation. The increased cardiometabolic risk in PCOS translates into a higher risk of COVID-19 complications, thus defining a distinct subgroup of women at increased risk of adverse COVID-19-related outcomes.

\section{Conclusion}

An extremely complex framework and additional obesity-related clinical features (such as tachypnea, minor lung capacity, reduced chest wall compliance, and aberrant respiratory muscle adaptations) may lead to severe respiratory failure requiring mechanical ventilation in obese COVID-19 patients. The low-grade chronic inflammatory state in obese people, as indicated by raised baseline serum levels of CRP, TNF- $\alpha$, and IL- 6 (positively correlated with BMI, waist circumference., and visceral adipose tissue), is responsible for initiating the cytokine storm in COVID-19 patients and can determine numerous underlying pathological situations and complications. Adipose tissue contains not only adipocytes (with significant intrinsic inflammatory properties) but also monocytes, macrophages, vascular components, and other cells contributing to inflammation. Additionally, adipocytes express many receptors that are sensitized by infectious agents, thus activating cytokine-mediated signal transduction cascades and releasing pro-inflammatory cytokines and acute phase reactants. In this established association between BMI-based obesity and severe course of COVID-19, body fat distribution seems to be crucial, with visceral adipose tissue significantly raising the possibility of a more severe course of COVID-19. The recog- nized interrelation between obesity and severe cases of COVID-19 supports the importance of instruments for risk assessment, not only BMI but also evaluation of waist-to-height ratio, waist circumference, and body composition with bioelectrical impedance analysis (which can evaluate lean and fat mass in physiologic and pathological conditions) [45, 46]: a quantification of visceral adipose tissue and upper abdominal circumference could help to phenotype and stratify younger individuals with visceral obesity at higher risk of COVID-19 morbidity and mortality, thus representing a promising strategy for risk assessment in COVID-19 patients. People worldwide should be encouraged to improve their lifestyle in order to reduce risks both in the current and subsequent waves of COVID-19.

Additional therapeutic and nutritional interventions focused on adipose tissue, such as medications/supplements contrasting the inflammatory state of obese patients, could decrease the risks of developing the severe form of COVID-19: obese individuals, as well as other subjects with abnormal body composition, could require tailored medical dietotherapy, in order to improve both short- and long-term COVID-19 outcomes.

\section{Acknowledgment}

The authors acknowledge the Department of Medical, Oral, and Biotechnological Sciences of University G. D'Annunzio for the support.

\section{Conflict of Interest Statement}

The authors declare no conflicts of interest.

\section{Funding Sources}

The research has not received external funding.

\section{Author Contributions}

M.A.G. wrote the draft and revised the final manuscript; N.D. supervised the project. 


\section{References}

1 Landecho MF, Marin-Oto M, RecaldeZamacona B, Bilbao I, Frühbeck G. Obesity as an adipose tissue dysfunction disease and a risk factor for infections-Covid-19 as a case study. Eur J Intern Med. 2021 Apr 2; S0953-6205(21):00097-2.

2 Sanchis-Gomar F, Lavie CJ, Mehra MR, Henry BM, Lippi G. Obesity and outcomes in COVID-19: when an epidemic and pandemic collide. Mayo Clin Proc. 2020;95: 1445-53.

3 Gkogkou E, Barnasas G, Vougas K, Trougakos IP. Expression profiling meta-analysis of ACE2 and TMPRSS2, the putative anti-inflammatory receptor and priming protease of SARS-CoV-2 in human cells, and identification of putative modulators. Redox Biol. 2020 Sep;36:101615.

4 Goossens GH, Dicker D, Farpour-Lambert NJ, Frühbeck G, Mullerova D, Woodward E, et al. Obesity and COVID-19: a perspective from the European Association for the study of obesity on immunological perturbations, therapeutic challenges, and opportunities in obesity. Obes Facts. 2020;13(4): 439-52.

5 deHeredia FP, Gómez-Martínez S, Marcos A. Obesity, inflammation and the immune system. Proc Nutr Soc. 2012;71:332-8.

6 Francisco V, Ruiz-Fernández C, Pino J, Mera A, González-Gay MA, Gómez R, et al. Adipokines: linking metabolic syndrome, the immune system, and arthritic diseases. Biochem Pharmacol. 2019;165:196-206.

7 Lelis D, de F, Freitas DF, de Machado AS, Crespo TS, Santos SHS. Angiotensin(1-7), adipokines and inflammation. Metab Clin Exp. 2019;95:36-45.

8 Landecho MF, Tuero C, Valentí V, Bilbao I, de la Higuera M, Frühbeck G. Relevance of leptin and other adipokines in obesityassociated cardiovascular risk. Nutrients. 2019;11(11):2664.

9 Gammone MA, D’Orazio N. Anti-obesity activity of the marine carotenoid fucoxanthin. Mar Drugs. 2015;13(4):2196-214.

10 Abella V, Scotece M, Conde J, Pino J, Gonzalez-Gay MA, Gómez-Reino JJ, et al. Leptin in the interplay of inflammation, metabolism and immune system disorders. Nat Rev Rheumatol. 2017;13:100-9.

11 deGit KCG, Peterse C, Beerens S, Luijendijk MCM, van der Plasse G, la Fleur SE, et al. Is leptin resistance the cause or the consequence of diet-induced obesity? Int J Obes. 2018;42(8):1445-57.

12 Cui H, López M, Rahmouni K. The cellular and molecular bases of leptin and ghrelin resistance in obesity. Nat Rev Endocrinol. 2017;13(6):338-51.

13 Fang H, Judd RL. Adiponectin regulation and function. Compr Physiol. 2018;8: 1031-63.
14 Ohashi K, Shibata R, Murohara T, Ouchi N. Role of anti-inflammatory adipokines in obesity-related diseases. Trends Endocrinol Metab. 2014;25(7):348-55.

15 Kumada M, Kihara S, Sumitsuji S, Kawamoto T, Matsumoto S, Ouchi N, et al. Association of hypoadiponectinemia with coronary artery disease in men. Arterioscler Thromb Vasc Biol. 2003;23:85.

16 Esposito K, Pontillo A, Di Palo C, Giugliano G, Masella M, Marfella R, et al. Effect of weight loss and lifestyle changes on vascular inflammatory markers in obese women: a randomized trial. JAMA. 2003;289(14): 1799-804.

17 Riccioni G, Gammone MA, Currenti W, D'Orazio N. Effectiveness and safety of dietetic supplementation of a new nutraceutical on lipid profile and serum inflammation biomarkers in hypercholesterolemic patients. Molecules. 2018;23(5):1168.

18 Gammone MA, Pluchinotta FR, Bergante S, Tettamanti G, D'Orazio N. Prevention of cardiovascular diseases with carotenoids. Front Biosci (Schol Ed). 2017 Jan 1;9:165-71.

19 Gammone MA, Riccioni G, Massari F, D’Orazio N. Beneficial effect of ivabradine against cardiovascular diseases. Front Biosci (Schol Ed). 2020 Mar 1;12:161-72. 161

20 Maury E, Brichard SM. Adipokine dysregulation, adipose tissue inflammation and metabolic syndrome. Mol Cell Endocrinol. 2010 Jan 15;314(1):1-16

21 Maury E, Noel L, Detry R, Brichard SM. In vitro hyper-responsiveness to TNF-\{alpha\} contributes to adipokine dysregulation in omental adipocytes of obese subjects. J Clin Endocrinol Metab. 2009 Mar 1;23(3):422.

22 Gammone MA, Riccioni G, D’Orazio N. Marine carotenoids against oxidative stress: effects on human health. Mar Drugs. 2015 Sep 30;13(10):6226-46.

23 Pasarica M, Sereda OR, Redman LM, Albarado DC, Hymel DT, Roan LE, et al. Reduced adipose tissue oxygenation in human obesity: evidence for rarefaction, macrophage chemotaxis, and inflammation without an angiogenic response. Diabetes. 2009;58:718-25.

24 Daryabor G, Kabelitz D, Kalantar K. An update on immune dysregulation in obesityrelated insulin resistance. Scand J Immunol. 2019;89:e12747.

25 Rodríguez A, Ezquerro S, Méndez-Giménez L, Becerril S, Frühbeck G. Revisiting the adipocyte: a model for integration of cytokine signaling in the regulation of energy metabolism. Am J Physiol Endocrinol Metab. 2015 Oct 15;309(8):E691-714.

26 Trayhurn P, Alomar SY. Oxygen deprivation and the cellular response to hypoxia in adipocytes-perspectives on white and brown adipose tissues in obesity. Front Endocrinol. 2015;6:19.
27 Sudhakar M, Silambanan S, Chandran AS, Prabhakaran AA, Ramakrishnan R. C-Reactive Protein (CRP) and leptin receptor in obesity: binding of monomeric CRP to leptin receptor. Front Immunol. 2018 May 29;9:1167.

28 Rashad NM, El-Shabrawy RM, Sabry HM, Fathy HA, Said D, Yousef MS. Interleukin- 6 and hs-CRP as early diagnostic biomarkers for obesity-related peripheral polyneuropathy in non-diabetic patients. Egypt J Immunol. 2018;25(2):153-65.

29 Park CH, Do JG, Lee YT, Yoon KJ. Sarcopenic obesity associated with high-sensitivity C-reactive protein in age and sex comparison: a two-center study in South Korea. BMJ Open. 2018;8(9):e021232.

30 Campbell CM, Kahwash R. Will complement inhibition be the new target in treating COVID-19-related systemic thrombosis? Circulation. 2020;141(22):1739-41.

31 Marongiu F, Grandone E, Barcellona D. Pulmonary thrombosis in 2019-nCoV pneumonia? J Thromb Haemost. 2020; 18(6):1511-3

32 Parra-Medina R, Herrera S, Mejia J. Systematic review of microthrombi in COVID-19 autopsies. Acta Haematol. 2021 Apr; 19:1-8.

33 Wichmann D, Sperhake JP, Lütgehetmann M. Autopsy findings and venous thromboembolism in patients with COVID-19: a prospective cohort study. Ann Intern Med. 2020;173(4):268-77.

34 Iba T, Levy JH, Levi M, Thachil J. Coagulopathy in COVID-19. J Thromb Haemost. 2020;18(9):2103-9.

35 Sengenes C, Miranville A, Lolmede K, Curat CA, Bouloumie A. The role of endothelial cells in inflamed adipose tissue. J Intern Med. 2007;262:415-21.

36 Alessi MC, Poggi M, Juhan-Vague I. Plasminogen activator inhibitor-1, adipose tissue and insulin resistance. Curr Opin Lipidol. 2007;18:240-5.

37 Bikdeli B, Madhavan MV, Gupta A, Jimenez D, Burton JR, Der Nigoghossian C, et al.; Global COVID-19 Thrombosis Collaborative Group. Pharmacological agents targeting thromboinflammation in COVID-19: review and implications for future research. Thromb Haemost. 2020;120(7): 1004-24.

38 Gammone MA, D’Orazio N. Obesity and COVID-19: a detrimental intersection. Front Endocrinol. 2021 Apr 30;12:652639.

39 Lighter J, Phillips M, Hochman S, Sterling S, Johnson D, Francois F, et al. Obesity in patients younger than 60 years is a risk factor for COVID-19 hospital admission. Clin Infect Dis. 2020;71(15):896-7. 
40 Cunningham JW, Vaduganathan M, Claggett BL, Jering KS, Bhatt AS, Rosenthal $\mathrm{N}$, et al. Clinical outcomes in young US adults hospitalized with COVID-19. JAMA Intern Med. 2020 Sep 9;181(3):379-81.

41 Simonnet A, Chetboun M, Poissy J, Raverdy V, Noulette J, Duhamel A, et al. High prevalence of obesity in severe acute respiratory syndrome coronavirus-2 (SARS-CoV-2) requiring invasive mechanical ventilation. Obesity. 2020;28(7):1195-9.
42 Malavazos AE, Secchi F, Basilico S, Capitanio G, Boveri S, Milani V, et al. Abdominal obesity phenotype is associated with COVID-19 chest X-ray severity score better than BMI-based obesity. Eat Weight Disord. 2021 Apr 5:1-15. Epub ahead of print.

43 Longmore DK, Miller JE, Bekkering S, Saner C, Mifsud E, Zhu Y, et al.; International BMI-COVID Consortium; International BMI-COVID Consortium. Diabetes and overweight/obesity are independent, nonadditive risk factors for in-hospital severity of COVID-19: an international, multicenter retrospective meta-analysis. Diabetes Care. 2021 Apr 15;44(6):1281-1290.
44 Subramanian A, Anand A, Adderley NJ, Okoth K, Toulis KA, Gokhale K, et al. Increased COVID-19 infections in women with polycystic ovary syndrome: a population-based study. Eur J Endocrinol. 2021 May;184(5):637-45.

45 Gammone MA, D’Orazio N. Assessment of body composition and nutritional risks in young ballet dancers-the bioelectrical impedance analysis. J Electr Bioimpedance. 2020;11(1):26-30.

46 Gammone MA, Ficoneri C, D’Orazio N. Assessment of body composition in oncologic patients: experimental survey on the role of bioimpedentiometric analysis. J Electr Bioimpedance. 2019;10(1):90-5. 